\title{
Triple Metachronous Malignancies with Thyroid Involvement: A Brief Overview of Five Case Reports over 20 Years of Institutional Experience
}

\author{
Marius-Ioan Bădan 1,*(D) and Doina Piciu 1,2 (D) \\ 1 Department of Morphological Sciences, Iuliu Hațieganu University of Medicine and Pharmacy, \\ 400012 Cluj-Napoca, Romania; doina.piciu@gmail.com \\ 2 Department of Endocrinology and Nuclear Medicine, "Prof. Dr. Ion Chiricuta" Institute of Oncology, \\ 400015 Cluj-Napoca, Romania \\ * Correspondence: badan_marius@yahoo.com; Tel.: +40-753426027
}

Received: 8 March 2020; Accepted: 17 March 2020; Published: 20 March 2020

\begin{abstract}
Multiple primary malignant tumors are defined by the development of more than one malignancy in a single patient. These can be subdivided into synchronous or metachronous malignant tumors, depending on their time of appearance, relative to the first malignancy. Triple primary malignancies are a relatively rare occurrence in day-to-day practice and triple synchronous or metachronous carcinomas involving a thyroid localization are even less common. In this case series, we report our 20-year experience in diagnosing and managing five patients with triple malignant tumors with thyroid involvement.
\end{abstract}

Keywords: triple primary malignant tumors; thyroid carcinoma; synchronous; metachronous

\section{Introduction}

First described in 1889 by Billroth [1] and later extensively reported on by Warren and Gates [2] in 1932, multiple primary malignant tumors (MPMT) are defined by two or more distinct malignancies occurring in the same patient. They are further subdivided into synchronous (the second malignancy is diagnosed within the first six months of the first primary tumor) or metachronous carcinomas (the second malignancy is diagnosed after six months). Emphasis should be given to not misidentifying synchronous cancers with metastases of histologically identical cancers diagnosed at different intervals [2].

Approximately thirty years after the Warren and Gates publication, it was becoming clear that an advanced age allowed for more cancers to appear over time [3]. As modern-day medicine expands our lifetime and the survival of cancer patients is prolonged, these types of synchronous and metachronous malignancies should not be disregarded as mere rare peculiarities. One seemingly extensive review of the literature reports a prevalence that varies between $0.73 \%$ and $11.7 \%$ [4]. Nevertheless, studies involving more than a hundred cases of MPMT demonstrate the increasing rarity of cases with more than two malignancies [5-8].

The most common cancers involved in MPMT belong to the digestive system, followed by lung, head, and neck carcinomas [7]. Although often described in association with lung and breast malignancies, MPMTs involving thyroid carcinomas are among the rarest tumors described [8-13].

In this case report series, we briefly describe five cases of MPMT involving thyroid carcinomas as part of triple metachronous associations.

\section{Methods}

We performed a 20-year retrospective analysis, between January 2000 and December 2019, of all cases of MPMT involving thyroid carcinomas within the "Prof. Dr. Ion Chiricuța" Institute of Oncology, 
Cluj-Napoca, Romania. In the last 20 years, among all 9227 cases of thyroid carcinoma from our evidence, synchronous or metachronous malignancies were present in 241 cases (2.61\%) and triple association of multiple malignancies involving thyroid was present in five cases, representing $0.05 \%$, 3 women and 2 males. The cases are presented in such a way as to not disclose any information which would lead to the identification of the person involved. The cases are described and analyzed in the following sections in decreasing order since their last admittance to our institute.

All patients signed a written consent during admission at our institute regarding their permission to process their data for research purposes.

\section{Case Presentations}

\subsection{Case Presentation Nr. 1}

A 54-year-old Caucasian female patient with a history of high grade bilateral ovarian carcinoma Silverberg II (pT1cNxM0L1V0), with a later worsened condition (ypT3aN0MxR0V0), was investigated by computed tomography (CT) for follow-up. A thoraco-abdominopelvic CT was performed revealing a large, compressive inhomogeneous nodule in the right thyroid lobe as well as a $37 \mathrm{~mm}$ pulmonary nodule in the left superior lung of uncertain malignant potential, besides enlarged mediastinal lymph nodes, ascites, and carcinomatosis. Even though the marker for differentiated thyroid cancer, thyroglobulin ( $\mathrm{Tg}$ ), was not indicated for the differential diagnosis, it was determined and showed elevated levels $(\mathrm{Tg}=260 \mathrm{ng} / \mathrm{mL} ; \mathrm{N} . \mathrm{V} .=3.5-77 \mathrm{ng} / \mathrm{mL})$. Thyroid echography revealed a completely nodular right thyroid lobule, which was increased in size (4 cm in greatest dimension), with multiple areas of calcification, intense vascularization, and an area of central necrosis. Scintigraphy with Tc-99m pertechnetate was performed describing a hot thyroid nodule, favoring a conclusion of adenoma (Figure 1).

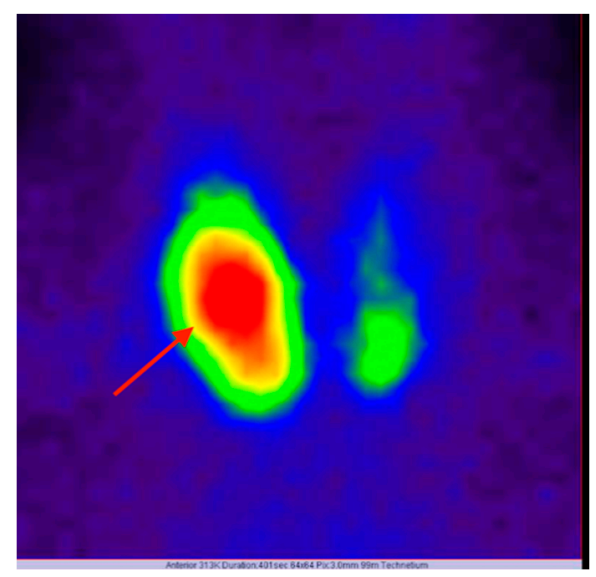

Figure 1. Thyroid scintigraphy with Tc-99m pertechnetate AP incidence showing a hot nodule (red arrow) in the right thyroid lobe, suggestive for thyroid adenoma (GE gamma camera LEHR); final histology exam being a hobnail variant of thyroid papillary microcarcinoma within an adenomatous nodule.

The fine-needle aspiration biopsy (FNAB) was refused. The patient was scheduled for reevaluation and began chemotherapy treatment with Paclitaxel $175 \mathrm{mg} / \mathrm{sqm}$ and Carboplatin 5AUC d1, q21d for four cycles. A subsequent evaluation of the thyroid nodule revealed a toxic nodule, with a low level of TSH (0.07 mIU/L; N.V. = 04-4.5 mIU/L) and the patient started the treatment with $10 \mathrm{mg}$ of Thyrozol and $10 \mathrm{mg}$ of Propranolol per day. CT follow-up revealed additional lesions located at the spine (L4 and D3) with the possibility of metastases, confirmed later on by scintigraphy with 99mTc-hydroxydiphosphonate (99mTc-HDP) and single-photon emission computed tomography (SPECT-CT). Total thyroidectomy was performed and later histopathological results revealed a 
$3 \mathrm{~mm}$ hobnail variant of thyroid papillary microcarcinoma (pT1aN0) within an adenomatous nodule (Figure 2).

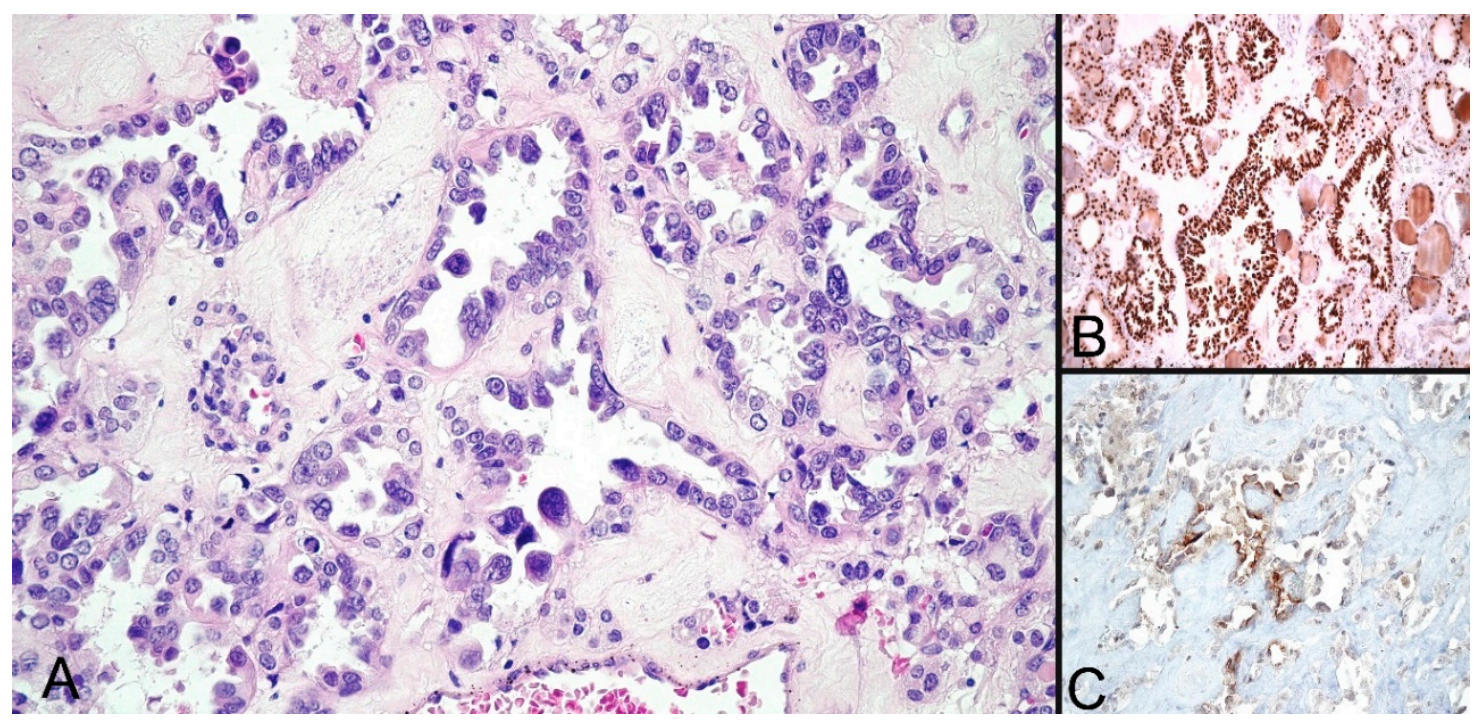

Figure 2. Hobnail variant of thyroid papillary carcinoma. (A) High power view (HPF) of hematoxylin-eosin (HE) staining showing hobnail appearance $(\times 400)$. (B) Immunohistochemical TTF-1 stain positivity $(\times 200)$. (C) Focal thyroglobulin immunohistochemical positivity of malignant cells $(\times 400)$. Both $(\mathbf{B}, \mathbf{C})$ immunohistochemistry $(\mathrm{IHC})$ markers were used to exclude other carcinomas with hobnail appearance.

Owing to a low-risk group stage I disease, according to guidelines [14], no radioiodine was indicated, and only thyroid hormone substitution was administered. After the intervention, the serum Tg level in TSH stimulated conditions (TSH $68 \mathrm{mIU} / \mathrm{L}$ ) was $<0.04 \mathrm{ng} / \mathrm{mL}$, with negative anti-Tg antibodies and negative cervical ultrasound, consistent with thyroid carcinoma complete remission. We performed a whole-body scan (WBS) with I-131 $370 \mathrm{MBq}$, which was negative, confirming that the lung and bone lesions were most likely not thyroid related. In these conditions, an F18-fluorodeoxyglucose (F18-FDG) PET/CT scan was performed, revealing a 35/30 mm pulmonary lesion with a pathological F18-FDG uptake of SUVlbm Max $=4.64$ and also an increased uptake in supposed bone metastases now located at L4, D12, D3, and D1 with an SUVlbm Max of 2.45 at D3. The patient underwent an endobronchial ultrasound-guided biopsy (EBUS-TBNA) and the diagnosis of ALK-negative pulmonary adenocarcinoma was revealed. Genetic studies uncovered an EGFR mutant status with c.2235_2249del deletion in exon 19, without the T790M mutation. The patient started treatment with Afatinib $40 \mathrm{mg}$ $1 \mathrm{tb}$ /day and continued to perform CT examination with a consistent diminishing size of the lung adenocarcinoma $(30 \mathrm{~cm})$ and a stable number of bone metastases. The last records of the patient showed continuing regular follow-ups, being alive at the moment of this report.

We underline the particularity of this case, regarding the first diagnosis of the thyroid nodule, incidentally discovered at a routine CT follow-up, even though the nodule was large and clinically detectable. Furthermore, we would like to highlight that in the clinical arena, there is a real necessity to adapt the protocols, according to different situational aspects; in this case, the refusal of an FNAB, the thyroglobulin determination in the initial evaluation, and the use of thyroid scintigraphy. The characteristic of a "hot nodule" was confusing because a malignancy in this situation is less frequent than in a "cold nodule". Moreover, there is a clear need to have histopathology reports whenever it is possible. Considering the awareness of two already known malignancies, the lung nodule would have been quite probably a metastasis, when in reality the third synchronous malignancy was revealed. 


\subsection{Case Presentation Nr. 2}

A 63-year-old Caucasian female patient with a history of subtotal thyroidectomy for a multinodular goiter underwent surgery for endometrial adenocarcinoma, following a worrying curettage carried out for abnormal bleeding, and received a total hysterectomy with bilateral adnexectomy. During the procedure, a $10 \mathrm{~cm}$ cyst, located on the left ovary, was removed. Frozen sections revealed a malignant proliferation. The final hematoxylin-eosin histopathological diagnosis was that of a synchronous endometrioid FIGO 2 carcinoma of the uterus alongside a serous papillary carcinoma arising in the left ovary.

Seven years later, the patient presented herself in our institute for the recurrence of the multinodular goiter. Ultrasonography demonstrated an 18.6/24.8 mm posterior, ill-defined, heterogeneous nodule with microcalcifications and Doppler vascular signal in and around the nodule. A second nodule was seen in the vicinity measuring $8 / 7.2 \mathrm{~mm}$ with a hypoechogenic halo and no Doppler signal. The patient underwent surgery for the remaining thyroid tissue and the biological material was sent for histopathological evaluation (Figure 3). The result was that of an atypical oxyphilic adenoma localized in the left remaining thyroid tissue, which also developed chronic lymphocytic thyroiditis. Subsequent immunohistochemistry (IHC) showed positivity for CK19 (Figure 3A), p53, and galectin3 (Figure 3D) on approximately $40 \%$ of the examined adenomatous surface and was negative for protein S100 and CEA with a variable Ki-67 between $6 \%$ and $18 \%$ (Figure $3 \mathrm{C}$ ). The IHC-stained cells were thus considered malignant in nature.

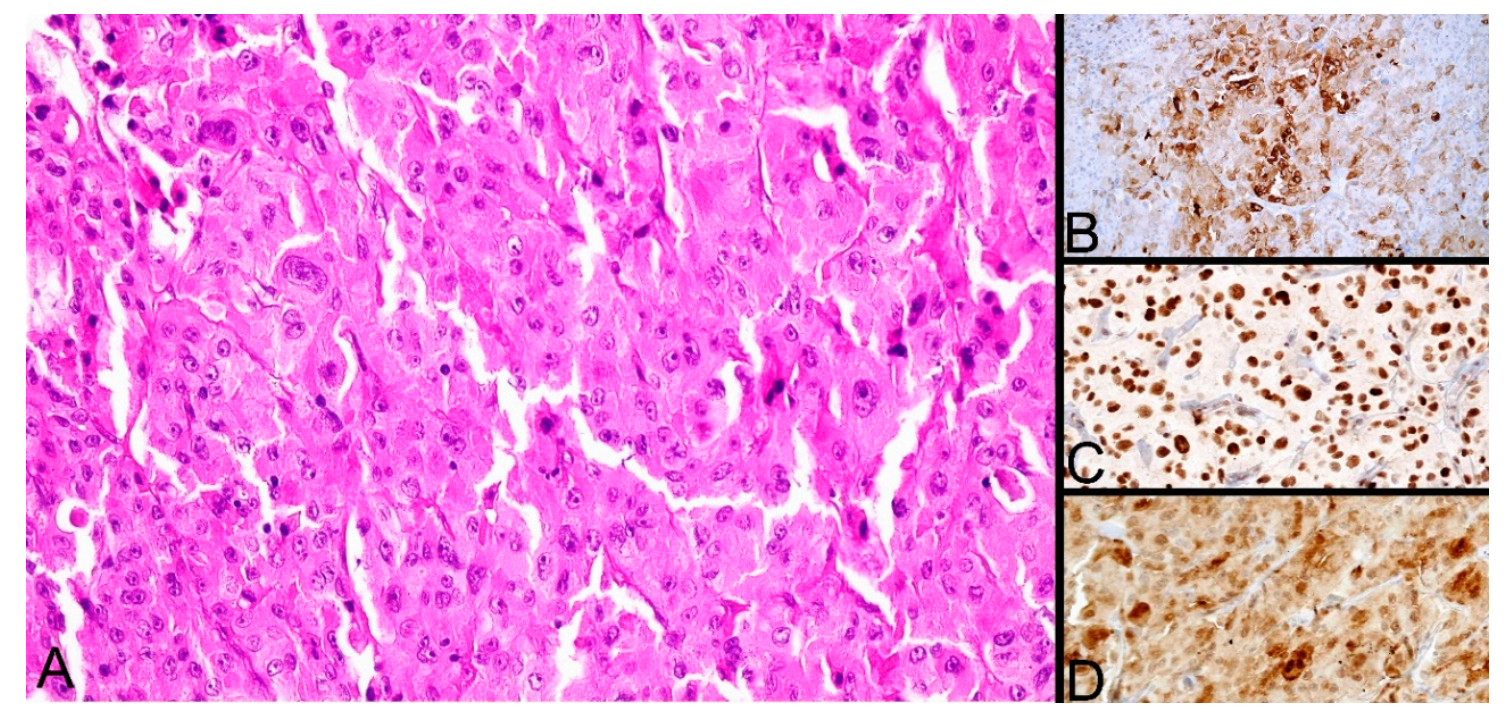

Figure 3. HE stain showing an oxyphilic adenoma. (A) Medium to large cells with abundant eosinophilic cytoplasm, characteristic for oxyphilic (Hürthle) cells, showing atypia $(\times 200)$. (B) Focal CK19 IHC positivity $(\times 200)$. (C) The Ki-67 (MIB-1) IHC marker for proliferation staining cell nuclei $(\times 400)$. (D) Galectin3 cell nuclear and faint cytoplasmic positivity $(\times 400)$.

The case is suggestive because 20 years ago the indication for surgery in thyroid nodules was more frequent and easily recommended, with a less precise preoperative characterization of the malignant potential, and the indication of subtotal thyroidectomy was relatively common. Moreover, this case is representative of a very unusual and extremely rare association of a Hürthle cell (oxyphilic) variant of follicular thyroid malignancy in association with two other malignancies in the same patient.

\subsection{Case Presentation Nr. 3}

A 58-year-old Caucasian male patient with a known history of papillary thyroid carcinoma with local regional lymph node metastases was surgically treated with a total thyroidectomy; subsequently, radioiodine therapy with a total activity of $5.4 \mathrm{GBq}$ was administrated. Hormone suppressive therapy 
and strict follow-ups, consisting of cervical ultrasounds, Tg and anti-Tg determined under stimulated conditions, as well as thoracal CTs and F18-FDG PET-CTs were done in the following years. In 2009, we performed an F18-FDG PET-CT scan revealing a $12 \mathrm{~mm}$ area with a moderate uptake of F18-FDG alongside a $45 \mathrm{~mm}$ nodular lesion, interpreted as an accessory spleen. Gastroscopy gave negative results. Later on, in 2014, the patient performed a thorax-abdominal CT scan, describing a $46 \mathrm{~mm}$ solid mass located within the greater curvature of the stomach next to an accessory spleen. Surgery was performed following this investigation revealing an approximately $3-4 \mathrm{~cm}$ polypoid tumor at the recto-sigmoid junction and a 5-6 cm subserous mass located on the posterior wall of the stomach. The following histopathological diagnosis was that of a synchronous moderately differentiated (G2) colo-rectal adenocarcinoma (pT3N2aMxL1V0, stage III B) and a gastrointestinal stromal tumor (GIST). The tumor presented as a moderately cellular mass (Figure 4) with a low mitotic index, not usually considered of high risk, but due to the initial size $(45 \mathrm{~mm}$ ) and its increases (up to $60 \mathrm{~mm}$ ) in the relatively short period of time, the tumor was regarded as having a relatively high malignant potential.

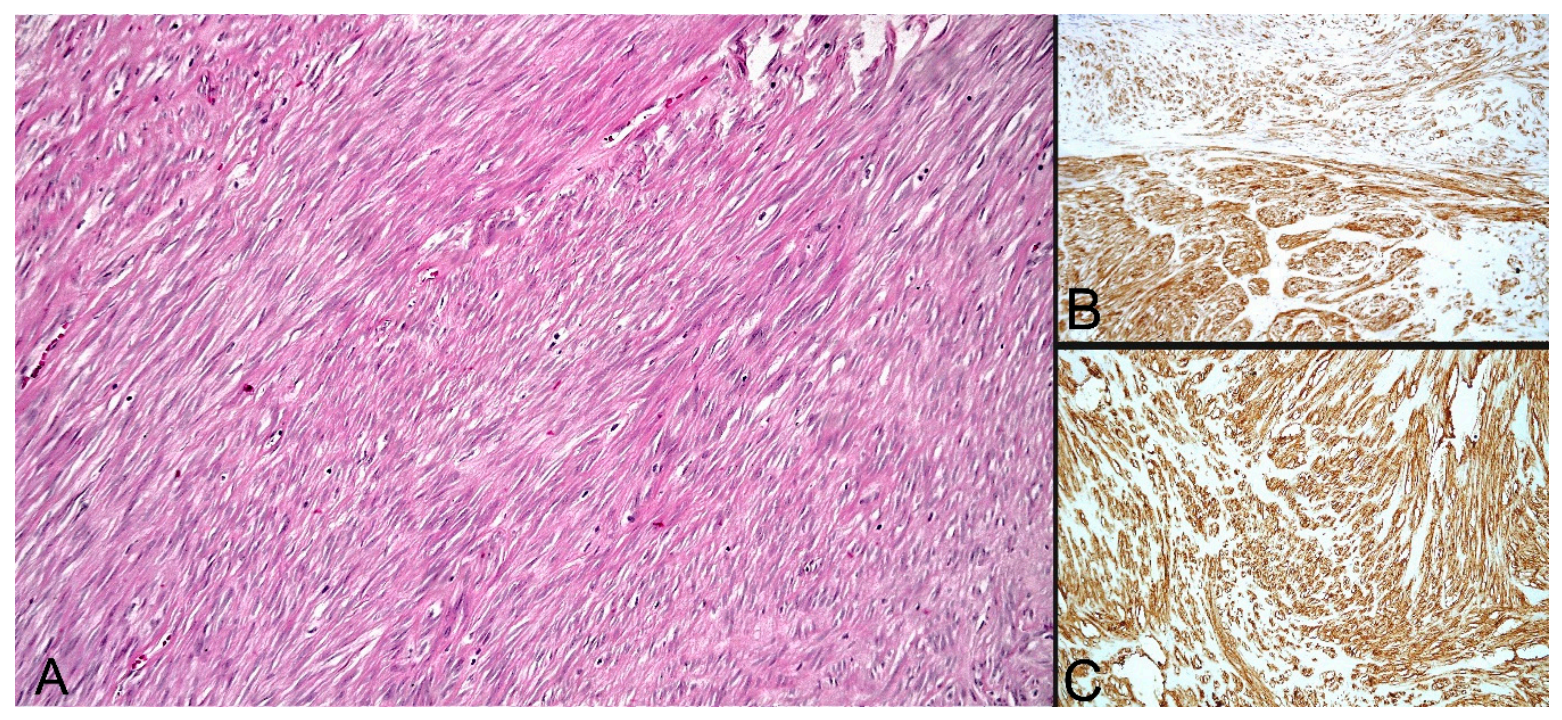

Figure 4. (A) HE stain of the gastro-intestinal stromal tumor with intertwining broad fascicles of spindle cells (×200). (B) IHC positivity for c-KIT (CD117) (×200). (C) CD34 IHC positivity (×200).

After the intervention, the patient was given adjuvant chemotherapy with a XELOX regimen with no adjuvant treatment recommended for the GIST. The treatment was unsuccessful in curing the disease, and in 2015, the patient went through brain surgery for metastases. The patient received external radiotherapy (with a total dose of $31 \mathrm{~Gy} / 10 \mathrm{fr}$ ) and after two more cycles of XELOX treatment developed an allergic reaction and was switched to a FOLFOX 4 regimen (a combination of oxaliplatin, folinic acid, and 5-fluorouracil).

This case underlines the necessity of standard screenings for colorectal cancer by means of routine investigations for occult hemorrhages and colonoscopy, even if there is an already known neoplasia; we should not dismiss the probability of multiple cancers and overlook the opportunity of performing some diagnostic procedures during the protocols of follow-up, even though they are not specifically indicated in the initial diagnosis of other malignant tumors.

\subsection{Case Presentation Nr. 4}

A 52-year-old Caucasian female patient was admitted and investigated through ultrasonography for thyroid enlargement, receiving a diagnosis of multinodular goiter with a follow-up of a total thyroidectomy later on. The final histopathological evaluation of the thyroid tissue revealed a papillary thyroid microcarcinoma (Figure 5) alongside the confirmation of the clinical diagnosis. 


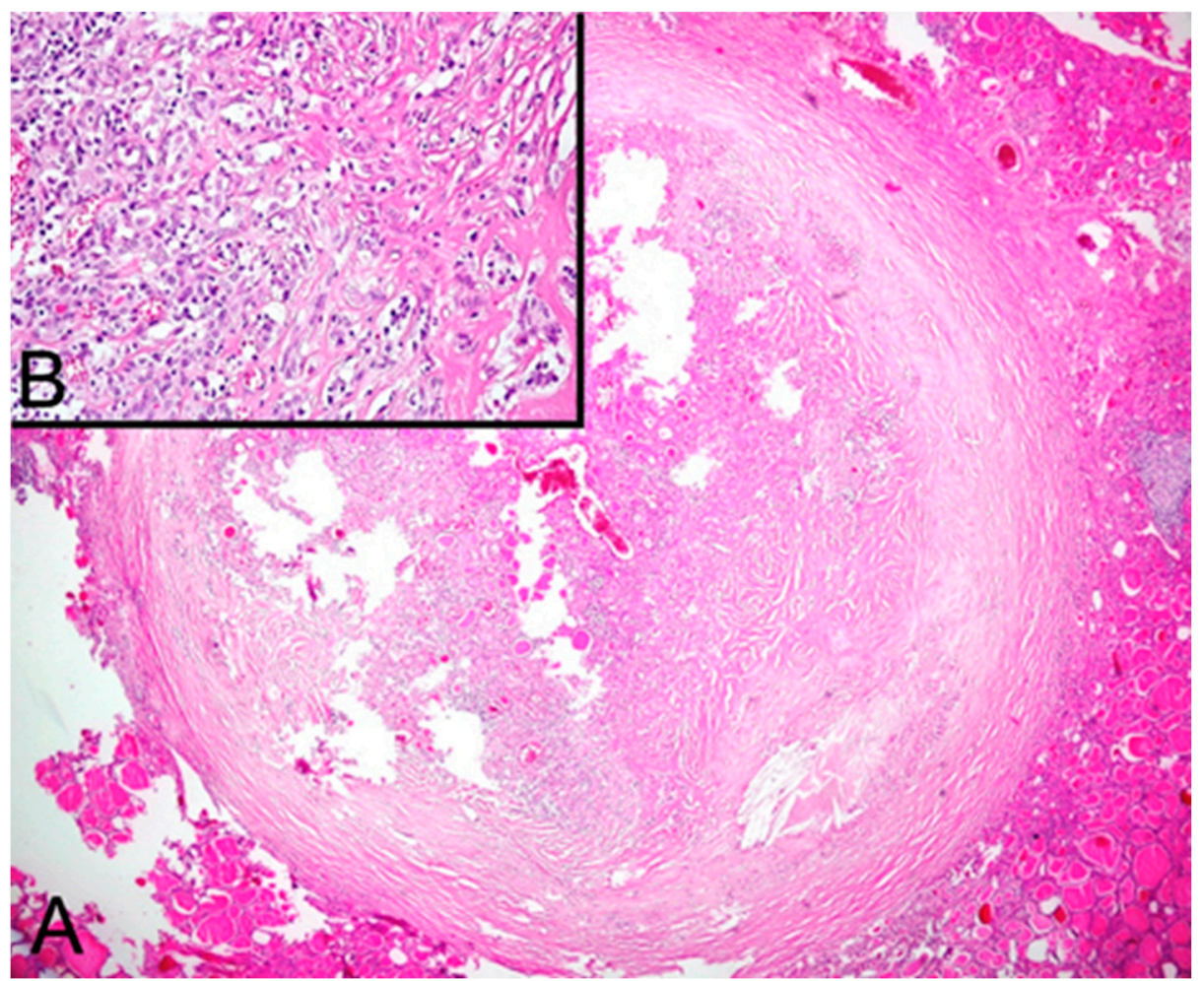

Figure 5. (A) HE stain showing a sclerosing-variant papillary thyroid carcinoma as a well-defined nodular mass $(\times 40)$. (B) HE high power field image of tumor cells within the mass $(\times 400)$.

The patient had a history of a recurrent vulvar keratinizing squamous cell carcinoma that was diagnosed and first operated nine years earlier (vulvectomy with right inguinal lymphadenectomy). Later on, after 7 years, the patient developed a uterine endometrioid carcinoma (FIGO 3, pT2a), which was surgically removed through a total hysterectomy with bilateral adnexectomy, and received adjuvant external radiotherapy (with a total dose of $50 \mathrm{~Gy}$ ). The patient underwent surgery, outside of the country, for a radical bilateral vulvectomy in 2010. Afterward, the same year, the patient was admitted into our institute for local relapse of the vulvar carcinoma. The following year, a CT scan was performed and a disease-free status was confirmed. The patient was last being treated for post-therapeutic myxedema, type 2 diabetes controlled through diet management, and second-degree hypertension.

This case underlines the necessity for whole-body evaluations in patients with a long history of malignancy in one area of the body. In such patients, we must not concentrate on the disease and its local reoccurrences and try to maintain a more holistic and open-minded approach to patient care. The thyroid microcarcinoma in our case was a serendipitous discovery.

\subsection{Case Presentation Nr. 5}

A 46-year-old Caucasian male patient was admitted in 2005 and surgically treated for a papillary thyroid carcinoma with a total thyroidectomy and selective lymphadenectomy (pT3N1aMx), followed by 3.7GBq I-131 and hormone suppressive therapy.

In 2007, an F18-FDG PET-CT scan was performed, which revealed a $30 / 28 \mathrm{~mm}$ focal increase of uptake in the sigmoid colon and a lesion of $21 \mathrm{~mm}$ in the VIIth liver segment, consisting of a colon malignant tumor with liver metastases (Figure 6). The patient was submitted for hemicolectomy and liver metastases resection, followed by chemotherapy. In 2013, during the routine examination, we found a focal uptake in the right prostatic lobe. After the prostatectomy, the histopathological result showed a prostate adenocarcinoma, occurring in a patient with already known thyroid and colon carcinomas (Figure 7). 


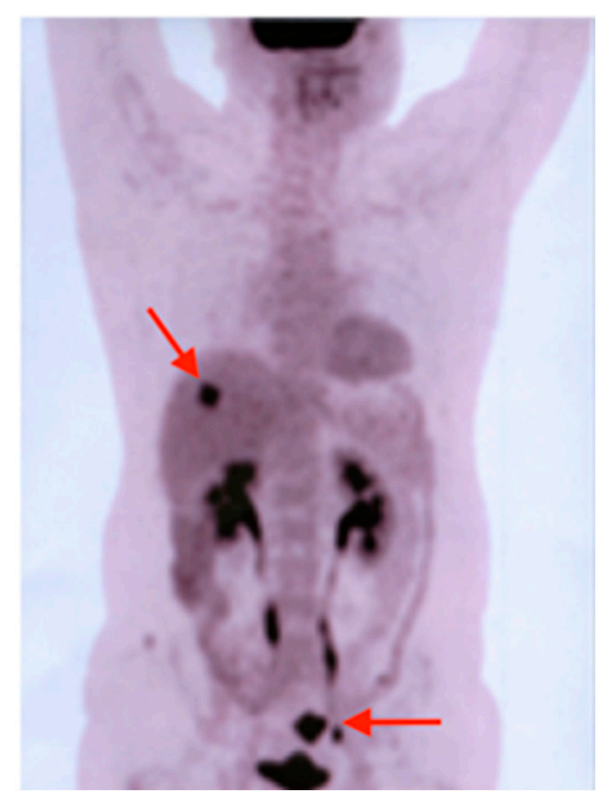

Figure 6. MIP image of F18-FDG PET-CT revealing a lesion in the sigmoid colon (lower-right red arrow) and a lesion of $21 \mathrm{~mm}$ in the VIIth liver segment (upper-left red arrow), consisting of a colon malignant tumor with liver metastases.

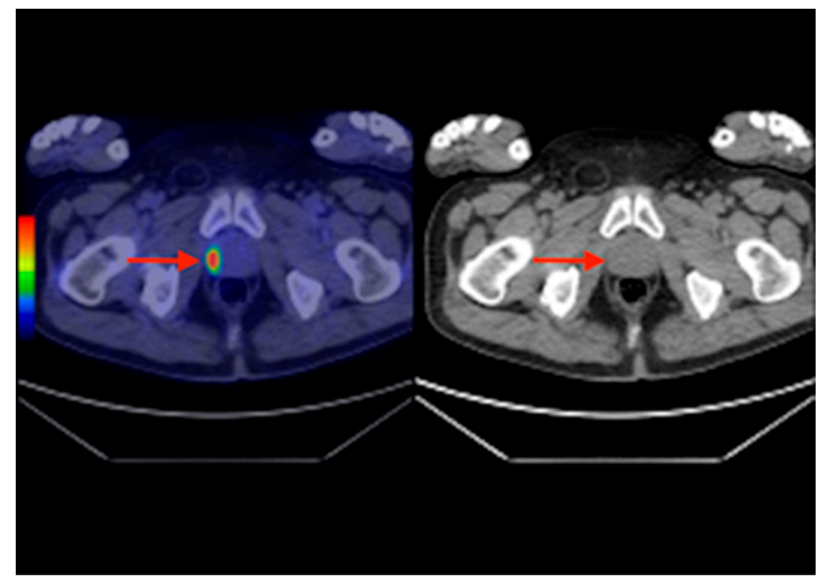

Figure 7. Axial section F18-FDG PET-CT (left) and CT (right) revealing an increased uptake in the right prostate lobe (red arrows) suggesting a primary prostate carcinoma, confirmed in histology.

This case underlines the important contribution of the F18-FDG PET-CT scan during the follow-up of malignant tumors.

\subsection{Overview}

A brief overview of the selected cases can be seen in the following table (Table 1):

Table 1. Summary of the cases.

\begin{tabular}{|c|c|c|c|c|c|c|}
\hline $\begin{array}{l}\text { Case } \\
\text { No. }\end{array}$ & $\begin{array}{l}\text { Location of } \\
\text { Malignancy }\end{array}$ & Histology & Gender & $\begin{array}{l}\text { Age at Thyroid } \\
\text { Carcinoma } \\
\text { Diagnosis }\end{array}$ & $\begin{array}{l}\text { Initial } \\
\text { Diagnosis } \\
\text { (Year) }\end{array}$ & $\begin{array}{c}\text { Disease-Free } \\
\text { Interval } \\
\text { (Years) }\end{array}$ \\
\hline \multirow{3}{*}{ Case 1} & ovarian & serous adenocarcinoma & \multirow{3}{*}{ female } & \multirow{3}{*}{53 years } & 2017 & NA \\
\hline & thyroid & papillary microcarcinoma & & & 2018 & 1 \\
\hline & lung & acinar adenocarcinoma & & & 2019 & 1 \\
\hline
\end{tabular}


Table 1. Cont.

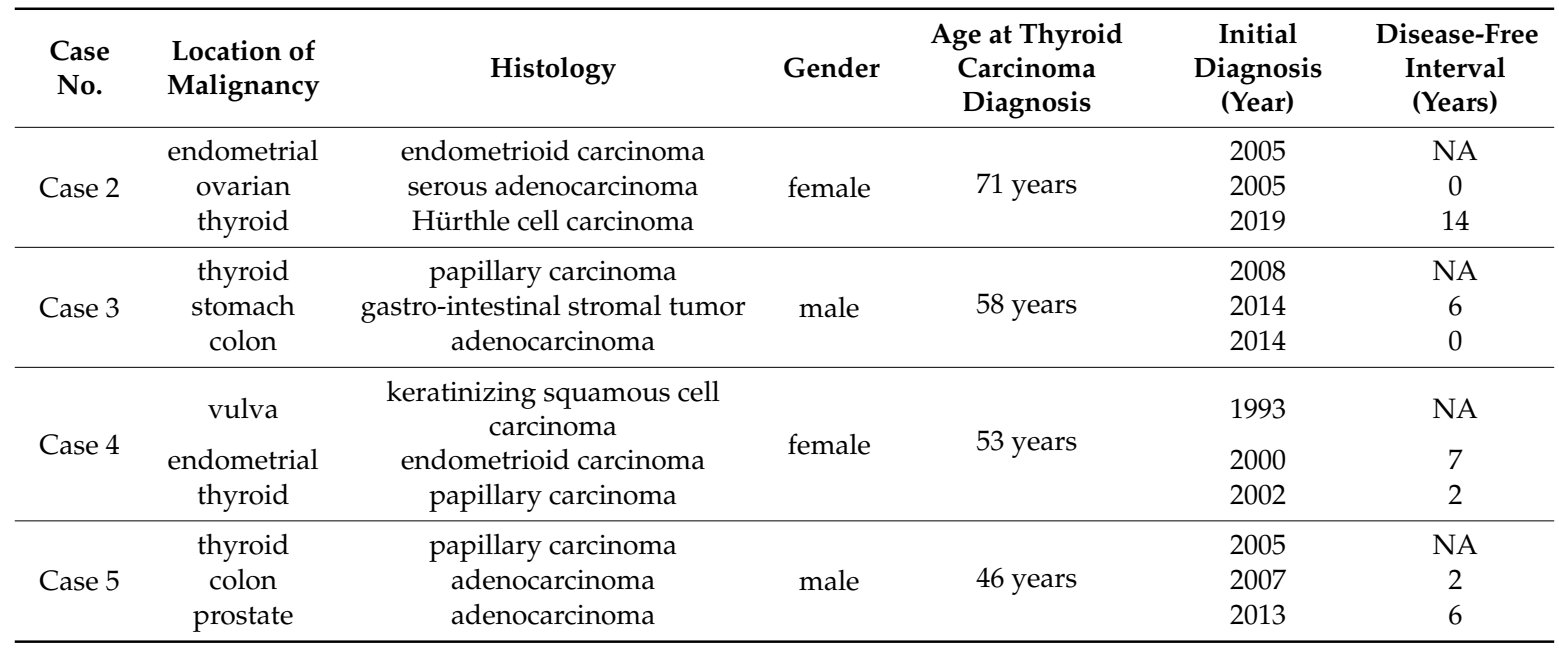

*NA = not applicable; no prior known malignancy.

\section{Discussions}

Our study aimed to describe triple MPMTs with thyroid involvement as a single-center experience. Other institute-level screenings have been conducted regarding MPMTs with double and triple cancer associations but they were not concentrated on triple thyroid-related malignancies $[9,15,16]$. Moreover, several case reports can be found in the scientific literature describing associations between thyroid carcinomas and at least two other malignancies in the same patients [17-19]. Somewhat unusual associations have been described between thyroid carcinomas and renal cell carcinoma or heart leiomyosarcomas [20,21]. Our search for relevant articles concerning triple cancer associations with thyroid involvement showed mostly single case reports and can be summarized by year of appearance and location of the second and third cancer in Table 2.

Table 2. Summary of Pubmed indexed articles of triple malignancies with thyroid involvement.

\begin{tabular}{ccc}
\hline Investigators & Year of Publication & $\begin{array}{c}\text { Location of Second and Third Malignancies } \\
\text { besides the Thyroid Carcinoma }\end{array}$ \\
\hline Peng et al. [22] & 2019 & Colon; Kidney \\
\hline Peng et al. [23] & 2018 & Lung; Kidney \\
\hline Kikuchi et al. [24] & 2017 & Stomach; Breast \\
\hline Singh et al. [25] & 2016 & Larynx; Lymph nodes \\
\hline Cohen et al. [26] & 2016 & Kidney; Skin \\
\hline Oh et al. [27] & 2015 & Stomach; Kidney \\
\hline Adams and Caffrey [28] & 2014 & Larynx; Thyroid (second) \\
\hline Lee et al. [17] & 2010 & Rectum; Uterus \\
\hline Omür et al. [29] & 2008 & Larynx; Lung \\
\hline Iqbal et al. [18] & 2008 & Kidney; Prostate \\
\hline Rai et al. [21] & 2007 & Lung; Stomach \\
\hline Hamada et al. [19] & 2000 & Heart; Lung \\
\hline Fukuoka et al. [20] & 2000 & Stomach; Rectum \\
\hline Shibutani et al. [30] & 1994 & Uterus; Lung \\
\hline Arimura et al. [31] & 1989 & \\
\hline
\end{tabular}


Table 2. Cont.

\begin{tabular}{ccc}
\hline Investigators & Year of Publication & $\begin{array}{c}\text { Location of Second and Third Malignancies } \\
\text { besides the Thyroid Carcinoma }\end{array}$ \\
\hline Ohi et al. [32] & 1988 & Skin; Brain \\
\hline Watanabe et al. [33] & 1984 & not specified \\
\hline Yamamoto et al. [34] & 1983 & Ovary; Brain \\
\hline Nemoto et al. [35] & 1977 & Kidney; Nasopharynx \\
\hline Ishii et al. [36] & 1977 & Stomach; Stomach \\
\hline
\end{tabular}

Our approach to describe these cases has been more focused on the medical imaging side of the diagnosis with a histopathology confirmation, rather than on biological markers and clinical symptoms. The importance of PET-CT imaging should be underlined as two of our cases had additional cancers discovered by routine PET-CT screenings for their initial diagnostic. This approach is more in line with current trends of relying more on imaging data [27] complemented with pathology reports. One such example is the case of combining different imaging techniques with dual radiopharmaceuticals in multiple carcinoma diagnostics for efficient evaluation [37]. More evidence in this regard is provided by Ishimori et al. in a study concerning 1912 patients who underwent whole-body PET-CT scans. A high F18-FDG uptake, suggestive for new, undiagnosed malignancies, was found in $79(4.1 \%)$ cases. Thyroid histopathological proven malignancies were found in six cases (second most common after lung) [38]. Unexpected F18-FDG uptake has been previously described particularly for lesions regarding the gastro-intestinal tract, leading to the discovery of malignant or premalignant proliferations [39]. Lastly, the importance of PET-CT hybrid imaging in diagnosing MPMTs is shown by Even-Sapir et al. who described 44 new malignancies found in 115 patients who underwent PET-CT evaluation for suspected second primary malignancies [40]. Following these recent publications, coupled with our own findings, it seems reasonable to consider that follow-up PET-CT screenings play a major role in the diagnostics of more than one primary malignancy in known cancer patients. Serum biomarkers are also potentially helpful and non-irradiating but lack the organ-specificity of hybrid imaging techniques. Several cancer biomarkers present elevated levels in more than one type of cancer, suggestive rather than diagnostic for a second primary malignancy, ultimately leading to further investigations through imaging techniques.

Even though our institute's data show an increase in new cases of thyroid cancer each year (Figure 8), with 9227 total cases of thyroid malignancies in the past 20 years, triple multiple primary malignancies, one being a thyroid cancer, were very rare $(0.05 \%)$. Double MPMTs with thyroid involvement were more frequent with 241 reported cases $(2.61 \%)$.

With the exception of two cases, most of our patients were female with a median age of 53 at the time of the thyroid carcinoma diagnosis. This could explain why the most common associations of thyroid localized carcinomas were, in our case, represented by gynecological malignancies (two cases with endometrioid endometrial carcinoma and two with serous papillary ovarian carcinoma, one vulvar squamous carcinoma, and one cervical adenocarcinoma). Even though Piciu et al. [14] showed an increased relative risk of developing a breast, uterine, or ovarian cancer after a primary differentiated thyroid carcinoma, in our case series, thyroid carcinomas developed after at least one gynecological carcinoma in all female patients. If we are looking for two malignancies, the most frequent association of MPMT in our institute was thyroid carcinoma and breast cancer, but in the association of triple cancers, this commune presentation was completely missing. Despite the fact that in larger populations the sex distribution seems to be more evenly spread [9], a female predominance with a similar age range has been previously reported and could be one of the reasons why papillary thyroid carcinomas seem to be more likely associated with breast malignancies [41]. Even though none of our cases featured breast malignancies, this association has been debated in the past since the 1960s [42]. Another possible explanation for this association could be that thyroid carcinomas are more 
prevalent in women $[43,44]$. Corso et al. [11] described a higher risk of developing a secondary thyroid malignancy in breast cancer patients who underwent hormonal therapy. An explanation could be the estrogen receptor that seems to be involved in both of the mechanisms that drive their appearance [12]. Attempts to characterize breast and thyroid tumors in order of emergence have not led to conclusive results, with an equal distribution in eight cases, but the widespread association of these malignancies suggests the importance of establishing a screening program for patients suffering from either of the primary diseases [13].

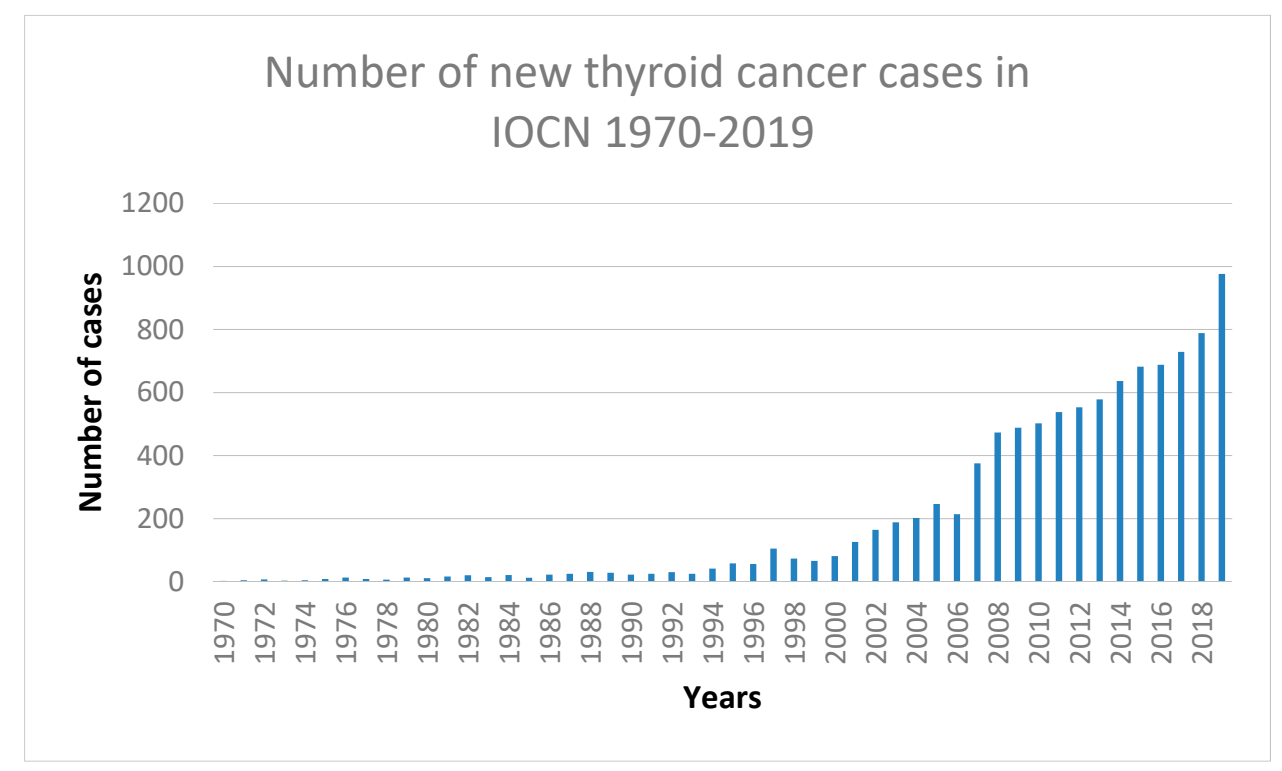

Figure 8. Graphical representation of thyroid cancer cases between 1970 and 2019 in the "Prof. Dr. Ion Chiricuța" Institute of Oncology (IOCN), Cluj-Napoca, Romania.

In our case series, except for Case Nr. 2, triple metachronous cancer associations seem to develop in a relatively short period of time from one to the other (Table 1). No obvious pattern of the disease-free interval was observed from our study samples. The most common histological type of thyroid malignancy was that of papillary thyroid carcinoma. An ample retrospective study performed in our institute showed a good 10-year survival $(80.4 \%)$ of patients diagnosed and treated with this type of pathology [44]. Two of our cases presented with synchronous malignancies, none of which were thyroid carcinoma associations. Although not thyroid-related, similar findings have been also reported by Kapoor et al. [45], with two months and three years of disease-free intervals. In some studies, nonetheless, more metachronous MPMTs were to be expected as they have been previously reported to be more common than synchronous tumors $[9,16]$.

Although the patient reviewed in our first case never received a biopsy confirmation of the bone metastases, they were most likely of pulmonary origin considering the extension and severity of the presentation. Nevertheless, in our experience, as well as in best practice, it is never a wrong decision to consider even the most remote of possibilities when dealing with differential diagnoses for bone metastases in a former thyroid carcinoma patient [46].

It would be noteworthy to study different types of common oncoprotein expressions in these carcinomas to see if there is any chance of syndrome-like associations. With regards to our fourth case presentation, Shibutani et al. [30] described a similar case of malignancy association and found both of the proteins expressed in the thyroid as well as in the rectal cancer tissue after performing IHC studies for p21 and p53. The p21 protein also stained surrounding adenomatous regions in the colon as well. Their variant of gastric malignancy (poorly differentiated adenocarcinoma) did not express the oncoproteins. In our case, a GIST would also be unlikely to express such proteins as shown by Ihle et al. [47]. Of course, others have also noted the association of thyroid and gastric carcinomas, 
as digestive MPMTs, alongside pulmonary neoplasms, have been reported to be more frequent [7], although contradicting data exists on this matter and seems to rely heavily on the study population [15]. Genetical studies in synchronous lung and thyroid carcinomas, in a triple MMPT case, showed that both of them carried EGFR and BRAF mutations but with distinct mutation profiles [23].

These types of association may not only have an important role in describing potential common pathways in pathogenesis but also, and more importantly, could be a way of applying a lesser number of targeted therapies in the future, as no definitive protocol currently exists on how to manage MPMTs from this point of view [48].

This study suffered from several identifiable limitations, some of which include the inadequate organization of the patient files, limited clinical data on symptoms and missing serum biomarkers in some cases as they were performed in tertiary private hospitals or institutions during long periods. Genetic or IHC studies for oncoproteins were not extensively utilized in the majority of our patients, consequently making in-depth analyses of the cases difficult to achieve. New 2017 World Health Organization modifications on papillary thyroid carcinomas (PTC) variants were taken into account. Although our study did not have follicular variants of papillary thyroid carcinomas, retrospective studies similar to ours could be influenced by the 2017 WHO modifications, as it has been shown to downgrade formerly PTC classified tumors by as much as $31 \%$ [49].

In conclusion, MPMTs should not be neglected in patients treated for a primary malignancy, even in the early years after treatment. Even within our limited sample size, we can observe that MPMTs can be detected through routine investigations (mostly through imaging techniques) even before early symptoms of the second malignancy. When dealing with female cancer patients, especially with breast cancer survivors or women diagnosed with gynecological carcinomas, care should be taken to screen for possible occult thyroid malignancies.

Author Contributions: Conceptualization, P.D. and B.M.-I.; writing-original draft preparation, B.M.-I.; writing-review \& editing, P.D. and B.M.-I.; investigation, B.M.-I.; P.D. provided conceptual input; supervision, P.D.; funding acquisition, B.M.-I. All authors have read and agreed to the published version of the manuscript.

Funding: The study was supported by Iuliu Hatieganu University of Medicine and Pharmacy, Cluj-Napoca, Romania (contract no. 1680/25/19.01.2018), based on Ph.D. contract no. 4115/01.10.2017.

Acknowledgments: This study was made possible by a collaboration with the "Prof. Dr. Ion Chiricuta" Institute of Oncology, Cluj-Napoca, Romania.

Conflicts of Interest: The authors declare no conflict of interest.

\section{References}

1. Billroth, T. Die Allgemeine Chirurgische Pathologie und Therapie in 51 Vorlesungen-Ein Handbuch fur Studierende und Arzte; Reimer, G., Ed.; Auflage: Berlin, Germany, 1889.

2. Warren, S.; Gates, O. Multiple primary malignant tumors: A survey of the literature and a statistical study. Am. J. Cancer 1932, 16, 1358-1414.

3. Spratt, J.S.; Hoag, M.G. Incidence of multiple primary cancers per man-year of follow up: 20-year review from the Ellis Fischel State Cancer Hospital. Ann. Surg. 1966, 164, 775-784. [CrossRef] [PubMed]

4. Demandante, C.G.N.; Troyer, D.A.; Miles, T.P. Multiple primary malignant neoplasms: Case report and a comprehensive review of the literature. Am. J. Clin. Oncol. 2003, 26, 79-83. [CrossRef] [PubMed]

5. Suzuki, T.; Takahashi, H.; Yao, K.; Inagi, K.; Nakayama, M.; Makoshi, T.; Nagai, H.; Okamoto, M. Multiple primary malignancies in the head and neck: A clinical review of 121 patients. Acta Otolaryngol. Suppl. 2002, 122, 88-92. [CrossRef]

6. Xu, L.L.; Gu, K.S. Clinical retrospective analysis of cases with multiple primary malignant neoplasms. Genet. Mol. Res. 2014, 13, 9271-9284. [CrossRef] [PubMed]

7. Zhai, C.; Cai, Y.; Lou, F.; Liu, Z.; Xie, J.; Zhou, X.; Zhanggui, W.; Fang, Y.; Pan, H.; Han, W. Multiple Primary Malignant Tumors-A Clinical Analysis of 15,321 Patients with Malignancies at a Single Center in China. J. Cancer 2018, 9, 2795-2801. [CrossRef] [PubMed] 
8. Li, F.; Zhong, W.-Z.; Niu, F.; Zhao, N.; Yang, J.-J.; Yan, H.-H.; Wu, Y.-L. Multiple primary malignancies involving lung cancer. BMC Cancer 2015, 15, 696. [CrossRef]

9. Gokyer, A.; Kostek, O.; Hacioglu, M.; Erdogan, B.; Kodaz, H.; Turkmen, E.; Hacibekiroglu, I.; Uzunoglu, S.; Cicin, I.; Osman, K.; et al. Clinical features of the patient with multiple primary tumors: Single center experience. North. Clin. Istanbul 2017, 4, 43-51. [CrossRef] [PubMed]

10. Lei, K.; He, X.; Yu, L.; Ni, C.; Chen, H.; Guan, D.; Sun, K.; Zou, H. Breast cancer prognosis is better in patients who develop subsequent metachronous thyroid cancer. PLoS ONE 2019, 14, e0215948. [CrossRef]

11. Corso, G.; Veronesi, P.; Santomauro, G.I.; Maisonneuve, P.; Morigi, C.; Peruzzotti, G.; Intra, M.; Sacchini, V.; Galimberti, V. Multiple primary non-breast tumors in breast cancer survivors. J. Cancer Res. Clin. Oncol. 2018, 144, 979-986. [CrossRef]

12. Yu, F.; Ma, J.; Huo, K.; Li, P. Association between breast cancer and thyroid cancer: A descriptive study. Transl. Cancer Res. 2017, 6, 393-401. [CrossRef]

13. Kim, J.H.; Kang, I.; Nam, S.; Park, H.S.; Park, S.; Jeong, J.J.; Nam, K.-H.; Chung, W.Y.; Kim, S.I.; Cho, Y.U.; et al. Comparison of characteristics in patients with both thyroid and breast cancer: Based on order of incidence. Korean J. Clin. Oncol. 2017, 13, 1-9. [CrossRef]

14. Piciu, D.; Pestean, C.; Barbus, E.; Larg, M.I.; Piciu, A. Second malignancies in patients with differentiated thyroid carcinoma treated with low and medium activities of radioactive I-131. Clujul Med. 2016, 89, 384-389. [CrossRef] [PubMed]

15. Irimie, A.; Achimas-Cadariu, P.; Burz, C.; Puscas, E. Multiple Primary Malignancies-Epidemiological Analysis at a Single Tertiary Institution. J. Gastrointest. Liver Dis. 2010, 19, 69-73.

16. Sharma, D.; Singh, G.; Kakkar, N.; Raj, S. Second primary malignancy: A retrospective analysis report from a tertiary cancer center of North India. Indian J. Cancer 2016, 53, 595. [CrossRef]

17. Lee, J.S.; Moon, W.; Park, S.J.; Park, M.I.; Kim, K.J.; Jang, L.L.; Park, M.J.; Chun, B.K. Triple synchronous primary cancers of rectum, thyroid, and uterine cervix detected during the workup for hematochezia. Intern. Med. 2010, 49, 1745-1747. [CrossRef]

18. Iqbal, F.R.W.; Sani, A.; Gendeh, B.S.; Aireen, I. Triple primary cancers of the larynx, lung and thyroid presenting in one patient. Med. J. Malays. 2008, 63, 417-418.

19. Hamada, Y.; Takise, A.; Uno, D.; Itoh, H.; Ichikawa, H.; Morishta, Y. Synchronous primary triple cancers including the lung, stomach, and thyroid: A case report. Kyobu Geka 2000, 53, 101-105.

20. Fukuoka, M.; Hagiwara, M.; Shimoshige, S.; Hirata, A.; Adachi, T.; Komura, H.; Shoji, T.; Kikuiri, T.; Ikeda, K.; Kimura, N.; et al. Primary leiomyosarcoma of the heart subsequent to double carcinomas of the thyroid and lung. Heart Vessels 2000, 15, 100-102. [CrossRef]

21. Rai, R.S.; Deb, P.; Rai, R.; Gupta, E.; Panayach, J.S. Synchronous primary triple neoplasia (renal cell carcinoma and prostate cancer in combination with thyroid neoplasm). Report of an unusual case. Minerva Urol. Nefrol. 2007, 59, 451-454.

22. Peng, C.; Li, Z.; Gao, H.; Zou, X.; Wang, X.; Zhou, C.; Niu, J. Synchronous primary sigmoid colon cancer and primary thyroid cancer followed by a malignant tumor of the kidney: Case report of multiple primary cancer and review of the literature. Oncol. Lett. 2019, 17, 2479-2484. [CrossRef] [PubMed]

23. Peng, L.; Zeng, Z.; Teng, X.; Chen, Z.; Lin, L.; Bao, H.; Shao, Y.W.; Wang, Y.; Dong, Y.; Zhao, Q. Genomic profiling of synchronous triple primary tumors of the lung, thyroid and kidney in a young female patient: A case report. Oncol. Lett. 2018, 16, 6089-6094. [CrossRef]

24. Kikuchi, N.; Ohashi, T.; Miura, T.; Nishibu, A.; Yamamoto, T. Triple cancers concurrently detected in a case of antitranscriptional intermediary factor- $1 \gamma$ antibody-positive dermatomyositis. Int. J. Dermatol. 2017, 56, 1516-1517. [CrossRef] [PubMed]

25. Singh, N.J.; Tripathy, N.; Roy, P.; Manikantan, K.; Arun, P. Simultaneous Triple Primary Head and Neck Malignancies: A Rare Case Report. Head Neck Pathol. 2016, 10, 233-236. [CrossRef] [PubMed]

26. Cohen, P.R. Segmental neurofibromatosis and cancer: Report of triple malignancy in a woman with mosaic Neurofibromatosis 1 and review of neoplasms in segmental neurofibromatosis. Dermatol. Online J. 2016, 22. pii: 13030/qt66k5j4wt.

27. Oh, S.J.; Bae, D.S.; Suh, B.J. Synchronous triple primary cancers occurring in the stomach, kidney, and thyroid. Ann. Surg. Treat Res. 2015, 88, 345-348. [CrossRef]

28. Adams, M.; Caffrey, R. Triple primary cancers of the head and neck: Case report and literature review. J. Laryngol. Otol. 2014, 128, 552-554. [CrossRef] 
29. Omür, O.; Ozcan, Z.; Yazici, B.; Akgün, A.; Oral, A.; Ozkiliç, H. Multiple primary tumors in differentiated thyroid carcinoma and relationship to thyroid cancer outcome. Endocr. J. 2008, 55, 365-372. [CrossRef]

30. Shibutani, Y.; Inoue, D.; Sugawa, H.; Mori, T. A case report of primary triple cancers in the thyroid, stomach and rectum with evidence of variable oncoprotein expressions. Nihon Naibunpi Gakkai Zasshi 1994, 70, 951-956.

31. Arimura, T.; Niwa, K.; Mitani, N.; Hagiwara, I.; Kawaida, T.; Shimazu, H. A resected case of triple cancer in the uterus, lung and thyroid. Nihon Kyobu Geka Gakkai Zasshi 1989, 37, 1233-1237.

32. Ohi, H.; Kikuchi, K.; Futawatari, K.; Kowada, M. A histologically-verified triple cancer-report of a rare case involving a primary brain tumor. Gan No Rinsho 1988, 34, 1001-1005.

33. Watanabe, S.; Kodama, T.; Shimosato, Y.; Arimoto, H.; Sugimura, T.; Suemasu, K.; Shiraishi, M. Multiple primary cancers in 5,456 autopsy cases in the National Cancer Center of Japan. J. Natl. Cancer Inst. 1984, 72, 1021-1027.

34. Yamamoto, Y.; Moriwaki, S.; Takashima, S.; Yumoto, Y. Autopsy case of simultaneous triple malignancies-ovarian, chordoma and thyroid cancers. Gan No Rinsho 1983, 29, 1375-1378. [PubMed]

35. Nemoto, R.; Shimizu, S.; Kato, T.; Tsuchida, S.; Takanashi, R.; Toda, T. Triple cancers consisting of renal cell carcinoma, epipharynx carcinoma, and thyroid carcinoma accompanied with primary hyperparathyroidism (author's transl). Nippon Hinyokika Gakkai Zasshi 1977, 68, 288-293. [PubMed]

36. Ishii, T.; Iri, H.; Yamamoto, S.; Sudoh, M. A triple cancer. Simultaneous occurrence of gastric carcinoid, adenocarcinoma and thyroid cancer. Am. J. Gastroenterol. 1977, 67, 171-176. [PubMed]

37. Søndergaard, E.; Ebbehoj, A.; Poulsen, P.L.; Gormsen, L.C. Multiple Neoplasms Simultaneously Diagnosed by Complementary Triple-Tracer PET/CT and 123I-MIBG Scintigraphy. Clin. Nucl. Med. 2017, 42, e61-e66. [CrossRef]

38. Ishimori, T.; Patel, P.V.; Wahl, R.L. Detection of unexpected additional primary malignancies with PET/CT. J. Nucl. Med. 2005, 46, 752-757.

39. Israel, O.; Yefremov, N.; Bar-Shalom, R.; Kagana, O.; Frenkel, A.; Keidar, Z.; Fischer, D. PET/CT detection of unexpected gastrointestinal foci of $18 \mathrm{~F}-\mathrm{FDG}$ uptake: Incidence, localization patterns, and clinical significance. J. Nucl. Med. 2005, 46, 758-762.

40. Even-Sapir, E.; Lerman, H.; Gutman, M.; Lievshitz, G.; Zuriel, L.; Polliack, A.; Inbar, M.; Metser, U. The presentation of malignant tumours and pre-malignant lesions incidentally found on PET-CT. Eur. J. Nucl. Med. Mol. Imaging 2006, 33, 541-552. [CrossRef]

41. Jena, A.; Patnayak, R.; Lakshmi, A.Y.; Manilal, B.; Reddy, M.K. Multiple primary cancers: An enigma. South Asian J. Cancer 2016, 5, 29. [CrossRef]

42. Chalstrey, L.J.; Benjamin, B. High incidence of breast cancer in thyroid cancer patients. Br. J. Cancer 1966, 20, 670-675. [CrossRef] [PubMed]

43. Rahbari, R.; Zhang, L.; Kebebew, E. Thyroid cancer gender disparity. Future Oncol. 2010, 6, 1771-1779. [CrossRef] [PubMed]

44. Piciu, D.; Irimie, A.; Piciu, A. Investigation of thyroid carcinoma over 40 years, using the database of the Ion Chiricuta Institute of Oncology Cluj-Napoca. J. BUON 2014, 19, 524-529. [PubMed]

45. Kapoor, A.; Narayan, S.; Nirban, R.; Purohit, R.; Bfagri, P.; Kumar, H.; Singhal, M. A rare case report of triple malignancy: Carcinoma urinary bladder, larynx, and breast in a single patient. Clin. Cancer Investig. J. 2014, 3, 558. [CrossRef]

46. Piciu, D.; Barbus, E.; Piciu, A.; Fetica, B. Mazabraud's syndrome and thyroid cancer, a very rare and confusing association: A case report. BMC Endocr. Disord. 2015, 15, 39. [CrossRef]

47. Ihle, M.A.; Huss, S.; Jeske, W.; Hartmann, W.; Merkelbach-Bruse, S.; Schildhaus, H.-U.; Büttner, R.; Sihto, H.; Sundby Hall, K.; Eriksson, M.; et al. Expression of cell cycle regulators and frequency of TP53 mutations in high risk gastrointestinal stromal tumors prior to adjuvant imatinib treatment. PLoS ONE 2018, 13, e0193048. [CrossRef]

48. Namal, E. Medical Treatment with Two Targeted Therapies for an Oncology Patient with Double Primary Cancer: Case Report. Turk. J. Oncol. 2016, 31, 51-54. [CrossRef] 
49. Borda, A.; Zahan, A.-E.; Piciu, D.; Barbuș, E.; Berger, N.; Nechifor-Boilă, A. A 15 year institutional experience of well-differentiated follicular cell-derived thyroid carcinomas; impact of the new 2017 TNM and WHO Classifications of Tumors of Endocrine Organs on the epidemiological trends and pathological characteristics. Endocrine 2019, 67, 630-642. [CrossRef] 